\title{
COMPARISON OF MULTIPHASIC CT SCAN OF ABDOMEN IN PATIENTS OF MESENTERIC ISCHAEMIA WITH SURGICAL FINDINGS OR CLINICAL FOLLOWUP
}

\author{
Amlendu Nagar1, Sheetal Singh2, Pramod Sakhi', Kumud Julka ${ }^{4}$, Anju Dhiman 5 \\ ${ }^{1}$ Associate Professor, Department of Radiodiagnosis, Index Medical College and Research Centre, Indore. \\ ${ }^{2}$ Associate Professor, Department of Radiodiagnosis, Index Medical College and Research Centre, Indore. \\ ${ }^{3}$ Professor, Department of Radiodiagnosis, Index Medical College and Research Centre, Indore. \\ ${ }^{4}$ Associate Professor, Department of Radiodiagnosis, Index Medical College and Research Centre, Indore. \\ ${ }^{5} 2^{\text {nd }}$ Year Junior Resident, Department of Radiodiagnosis, Index Medical College and Research Centre, Indore.
}

\section{ABSTRACT}

\section{BACKGROUND}

Acute and chronic mesenteric ischaemia remains a complex disease entity characterised by perfusion abnormality to the GI tract as it presents with nonspecific symptoms. MDCT offers high spatial resolution, fast scan times, 3D data sets, and excellent evaluation of nonvascular findings. Multiphasic CT scan features of acute bowel ischaemia will depend on its cause, location, extent and severity. Wide ranges of findings are seen in patients with mesenteric ischaemia. In fact, CT has demonstrated very high sensitivity and specificity for the diagnosis of mesenteric ischaemia and has replaced catheter angiography as the primary imaging modality of choice.

\section{MATERIALS AND METHODS}

We have retrospectively reviewed 37 cases of mesenteric ischaemia which were diagnosed by MDCT scan during 27 months and were further sent for surgery and or DSA and clinical followup. These patients were further grouped to acute and chronic depending on stenosis/status of mesenteric vessels, bowel wall changes and collateral pathways, and CT findings were correlated with surgical/DSA findings/followup.

\section{RESULTS}

Common findings of acute mesenteric ischaemia are Bowel wall thickening (80 \%), Bowel distention (53.3 \%) and altered bowel wall enhancement (46.6\%), while other findings SMA dissection, Pneumatosis and Pneumoporta were 33.3\%. Common findings of chronic mesenteric ischaemia are blocked SMA and collaterals filling post-block SMA (100\%), stenosis of superior mesenteric artery (77.3\%), stenosis of IMA (54.5\%), and stenosis of celiac trunk (31.8\%).

\section{CONCLUSION}

Multiphasic MDCT in mesenteric ischaemia offers excellent evaluation of vascular structures, bowel wall and demonstrates possible primary cause of mesenteric ischaemia.

\section{KEYWORDS}

Acute Mesenteric Ischaemia, Chronic Mesenteric Ischaemia, Multiphasic CT Scan, Surgical Findings. Thrombosis, Bowel Wall Enhancement, Pneumatosis.

HOW TO CITE THIS ARTICLE: Nagar A, Singh S, Sakhi P, et al. Comparison of multiphasic CT scan of abdomen in patients of mesenteric ischaemia with surgical findings or clinical followup. J. Evolution Med. Dent. Sci. 2017;6(28):2305-2310, DOI: $10.14260 /$ Jemds/2017/496

\section{BACKGROUND}

Acute and chronic mesenteric ischaemia remains a complex disease entity characterised by perfusion abnormality to the GI tract. Because it presents with nonspecific symptoms and laboratory findings, mesenteric ischaemia remains a clinical diagnostic challenge.

Acute mesenteric ischaemia is a circulation insufficiency event, occurring either suddenly or rapidly over a few weeks, that leads to ischaemic changes in bowel or other abdominal organs.

Financial or Other, Competing Interest: None.

Submission 13-01-2017, Peer Review 25-03-2017,

Acceptance 31-03-2017, Published 06-04-2017.

Corresponding Author:

Amlendu Nagar,

\#286, M. G. Road

Indore-452002,

India.

E-mail: dramlendu@gmail.com

DOI: $10.14260 /$ jemds $/ 2017 / 496$
Although mesenteric ischaemia occurs infrequently, the mortality rate is from 60 to $100 \%$, depending on the source of obstruction.1,2 The risk of atherosclerotic-related mesenteric occlusive disease increases with ageing and those who have severe cardiovascular disease are at the highest risk. 20 to $30 \%$ of hospital admissions for acute mesenteric ischaemia are from nonocclusive mesenteric ischaemia. ${ }^{3}$ Nearly 5 to $15 \%$ of all acute mesenteric ischaemic cases are from venous thrombosis. 4

The clinical features remain nonspecific, including abdominal pain, nausea, vomiting, and diarrhoea, all of which can overlap with other acute or chronic abdominal conditions. As a result, the diagnosis of MI remains a challenging clinical diagnosis. Imaging plays an important role in primary diagnosis of MI, and also aids in exclusion of other differential diagnostic considerations. MDCT offers high spatial resolution, fast scan times, 3D data sets, and excellent evaluation of nonvascular findings. In fact, CT has demonstrated very high sensitivity and specificity for the 
diagnosis of MI, and has replaced catheter angiography as the primary imaging modality of choice. ${ }^{5}$

\section{There are Three Stages of Acute Bowel Ischaemia-}

1. Stage 1- Mucosal necrosis and mucosal erosion or haemorrhage and it is reversible.

2. Stage 2- The damage extends to submucosal and muscular layers. ${ }^{6}$ Stages I and II are poorly identified on CT. Mucosal enhancement and wall thickening can be detected, but those findings are usually mild and nonspecific. ${ }^{6}$

3. Stage 3- Transmural bowel wall necrosis. The CT findings are more specific and reliable and display absent bowel wall enhancement, mesenteric strandings, ascites, pneumatosis and gas in portal veins. ${ }^{6}$

Chronic mesenteric ischaemia affects elderly individuals with history of atherosclerosis.6,7 The reason for mesenteric ischaemia is arterial occlusion due to atheroma. Usually proximal arterial segments are occluded in chronic mesenteric ischaemia. Non-atheromatous causes for occlusion are Takayasu arteritis, thromboangiitis obliterans and dysplastic lesions. ${ }^{7,8}$ Symptoms of bowel ischaemia depend on site and number of occlusion lesions, their chronicity and status of collateral vessels. 9,10

There are a few collateral pathways between the three main mesenteric vessels, which can be important for preserved downstream perfusion in the setting of proximal vascular narrowing or occlusion. The primary collateral flow between the celiac and SMA distributions is through the pancreaticoduodenal arcade, which connects the celiac axis via the common hepatic artery and gastroduodenal artery, to branches of the proximal SMA through a vascular plexus surrounding the head of pancreas and duodenal C-loop. Two potential routes of collateral flow are present between the SMA and IMA, including the marginal artery of Drummond that courses peripherally along the mesenteric margin/reflection of the colon, and the Arc of Riolan, which is located in the more central portions of the mesentery. ${ }^{11}$ In addition, there are connections from the mesenteric circulation to the deep pelvic arteries through a vascular plexus around the rectum. This allows communication of the IMA through the superior haemorrhoidal artery, to the branches of internal iliac arteries including the middle and inferior haemorrhoidal arteries.

MDCT examination is a comprehensive study for mesenteric ischaemia with detailed evaluation of mesenteric vessels, status of bowel wall and surrounding mesentery and extent and possible cause of mesenteric ischaemia. Angiography is considered the gold standard for the diagnosis of mesenteric ischaemia, but it is an invasive method, can rarely cause morbidity, and does not allow the evaluation of the bowel and other related findings. ${ }^{12-14}$

MDCT technology has dramatically improved the performance of CT scan which acquires rapid volumetric data over a large anatomic volume. This allows retrospective multiplanar reformatted images and CT angiograms with 2-D and 3-D visualisation. These advantages are helpful in identifying the site, level and cause of bowel ischaemia by showing abnormal segments of bowel, mesentery and vessels. In addition, other pathological conditions can also be detected in patients suspected of mesenteric ischaemia. The ability of modern MDCT for diagnosing mesenteric ischaemia has recently been reported to have a sensitivity of approximately $90 \% .12,15,16$

\section{Aims and objective}

The aim of the study is to retrospectively review patients of mesenteric ischaemia diagnosed by MDCT scan in our hospital and were further sent for surgery and or DSA during May 2014 to July 2016.

\section{MATERIALS AND METHODS}

A total of 37 patients were diagnosed as mesenteric ischaemia on MDCT findings during May 2014 to July 2016 at our institute. We have retrospectively analysed MDCT findings in these groups of patients. They were further grouped to acute (15 patients) and chronic (22 patients) depending on stenosis/status of mesenteric vessels, bowel wall changes and collateral pathways.

Acute mesenteric ischaemia of 15 patients were correlated with surgical findings, the sensitivity and specificity of each MDCT finding were assessed with surgical findings, while MDCTA findings of chronic mesenteric ischaemia of 22 patients were correlated with DSA findings and then subsequently their sensitivity and specificity were analysed.

All these patients had undergone MDCT Examination on 128 detector rows Siemens definition AS scanner using protocols as mentioned below in Table/Fig. 1. CT images were obtained from the dome of the liver to the level of the perineum to cover the entire course of the intestine. With MDCT scanners, a collimation of $0.625 \mathrm{~mm}$ and a detector pitch of 1.0-2.0 were used. Images with a 5 section thickness were usually constructed for interpretation; however, thinner sections of contiguous $1 \mathrm{~mm}$ were constructed for multiplanar image reformations and CT angiography. Sagittal images were obtained for assessing the origin of the mesenteric arteries and their variation. CT angio images were generated on Siemens Syngo.via work station.

\section{Statistical Method}

The obtained data was entered in Microsoft Excel 2016 for Windows. Frequencies (n) and percentages (\%) of variables were calculated. Results were obtained and tabulated using Microsoft Excel spreadsheet. Also, Sensitivity and Specificity were calculated. Data analyses were performed using version 17.0 of the Med Calc software program (MedCalc Software BVBA, Acacialaan, Ostend, Belgium).

\section{RESULTS}

\section{Acute Mesenteric Ischaemia}

Following findings were observed in our series in patients with acute mesenteric ischaemia and are mentioned below in Table/Fig. 2.

Sometimes thrombus or embolus is detected in peripheral branches. (Table/Fig. 3). The bowel wall thickening was a common finding observed in 12 (80\%) out of 15 patients of acute mesenteric ischaemia. Thickening of bowel wall is a common finding due to oedema and also haemorrhage. Normal small bowel thickness is 3 to $5 \mathrm{~mm}$ and mildly varies with degree of distension. (Table/Fig. 4). Dissection of arteries is seen in arterial phase and better appreciated on multiplanar reconstructions. (Table/Fig. 5). Bowel wall distension is seen due to interruption of peristalsis secondary to ischaemic insult and bowel wall necrosis. (Table/Fig. 6). Distension of bowel wall was seen in 
8 out of 15 patients of AMI. SMA dissection was seen in 5 $(33.3 \%)$ patients. Enhancement alterations in bowel wall is due to oedema and lack of enhancement is due to severe ischaemia and/or necrosis and that was seen in 8 out of 15 patients. Hyperaemia of bowel wall suggests viability of bowel segment. (Table/Fig. 4). Presence of air in bowel wall suggests pneumatosis, seen as a result of transmural bowel wall necrosis (Table/Fig. 7). Presence of air in mesenteric venous branches extending to liver in peripheral portal vein branches suggests pneumoporta. Pneumatosis was seen in 5 (33.3\%) patients while Pneumoporta was observed in 5 $(33.3 \%)$ patients. SMA thrombosis was seen in 2 patients (13.3\%). Associated other organ ischaemia (in kidney) was seen in 2 patients. The sensitivity and specificity of bowel wall thickening in correlation with surgical findings was 91.6 and $100 \%$ respectively, while specificity of pneumatosis and pneumoporta was 100 percent.

\section{Chronic Mesenteric Ischaemia}

MDCT findings in chronic mesenteric ischaemia are given in Table/Fig. 8.

Common findings of chronic mesenteric ischaemia are stenosis of celiac trunk (Table/Fig. 9), stenosis of superior mesenteric artery (Table/Fig. 10), blocked SMA and collaterals filling post-block SMA (Table/Fig. 11), stenosis of inferior mesenteric artery and filling of collateral vessels, pancreatic arch and marginal artery of colon. (Table/ Fig. 12, 13).

Stenosis of superior mesenteric artery is usually associated with chronic mesenteric ischaemia and was seen in 17 out of 22 patients (77\%). Stenosis of inferior mesenteric artery was seen in $12(54.5 \%)$ patients. Stenosis of celiac trunk and/or stenosis of inferior mesenteric artery is also commonly associated with SMA stenosis. Celiac artery stenosis was seen in 7 (31.81\%) patients. Usual collateral vessels are seen between celiac trunk and SMA via gastroduodenal artery and pancreatic arch. Pancreatic arch collaterals were seen in all 22 out of 22 patients of chronic mesenteric ischaemia. There are tortuous marginal arteries of the colon forming collateral pathways between SMA and IMA and were seen in all 22 patients of chronic mesenteric ischaemia. The sensitivity of SMA, IMA and celiac artery stenosis with DSA was $100 \%$, while the specificity was $83.3 \%$, $90.9 \%, 93.7 \%$ respectively. The sensitivity and specificity of collateral pathway-pancreatic arch \& marginal artery of the colon were 100 percent.

\begin{tabular}{|c|c|}
\hline Variables & Used Protocol \\
\hline Oral contrast & $\begin{array}{l}\text { Water (negative) or no oral contrast } \\
\text { so as to assess status of bowel wall. }\end{array}$ \\
\hline Intravenous Contrast & $\begin{array}{c}\text { Omnipaque } 300 \mathrm{mg} / \mathrm{mL} \text { of iodine@2 } \\
\text { mL per kg body weight injected at the } \\
\text { rate of } 4.5 \mathrm{~mL} / \mathrm{s} \text { using Pressure } \\
\text { injector. Followed by } 40 \mathrm{~mL} \text { normal } \\
\text { saline injection. }\end{array}$ \\
\hline Multiphasic Imaging & $\begin{array}{c}\text { Pre-injection non-contrast series } \\
\text { followed by bolus chasing arterial, } \\
\text { venous and delayed multiphasic } \\
\text { imaging }\end{array}$ \\
\hline Collimation & $64 \times 0.6 \mathrm{~mm}$ \\
\hline Helical pitch & 53 \\
\hline Gantry rotation time & 0.33 seconds \\
\hline $\mathrm{kV}$ & 120 \\
\hline $\mathrm{mA}$ & Automated low dose \\
\hline \multicolumn{2}{|c|}{$\begin{array}{l}\text { Table/Figure 1. CT Examination Protocol } \\
\text { for Multiphasic CT Scan of Abdomen }\end{array}$} \\
\hline
\end{tabular}

\begin{tabular}{|c|c|c|c|c|}
\hline CT Findings & $\begin{array}{c}\text { Acute mesenteric Ischaemia on } \\
\text { MDCT (15 patients) }\end{array}$ & $\begin{array}{c}\text { Surgical Finding } \\
\text { (15 patients) }\end{array}$ & Sensitivity (\%) & Specificity (\%) \\
\hline Bowel wall thickening & 12 & 11 & 91.6 & 100 \\
\hline Bowel distention & 8 & 7 & 58.3 & 66.6 \\
\hline Bowel wall non-enhancement & 7 & 6 & 50 & 66.6 \\
\hline SMA dissection & 5 & 4 & 33.3 & 66.6 \\
\hline Pneumatosis & 5 & 5 & 41.6 & 100 \\
\hline Pneumoporta & 5 & 5 & 41.6 & 100 \\
\hline SMA thrombosis & 2 & 2 & 8.3 & 66.6 \\
\hline
\end{tabular}

Table/Figure 2. MDCTA and Surgical Correlation in Patients with Acute Mesenteric Ischaemia

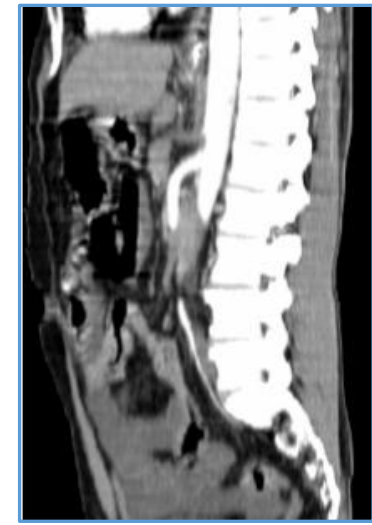

Table/Figure 3. Sagittal MPR Displaying Contrast opacified Aorta and Thrombosis in Mid-segment of Superior Mesenteric Artery in a Patient with Acute Bowel Ischaemia.

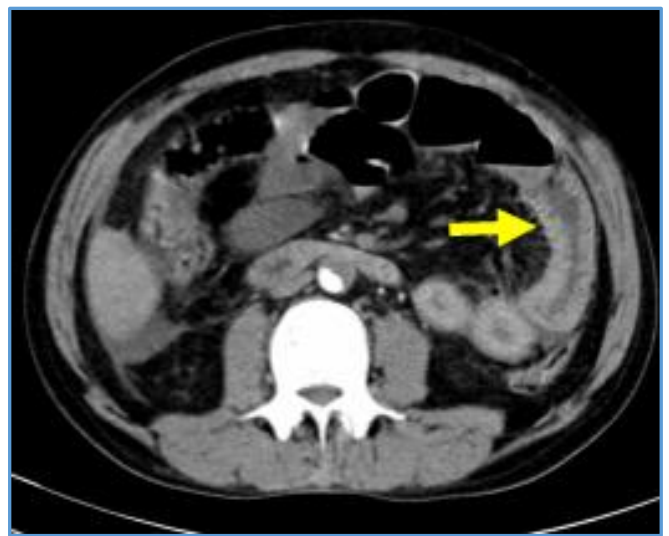

Table/Figure 4. Axial CT Image Showing Bowel Wall Thickening in Early Stages of Acute Bowel Ischaemia because of Submucosal Haemorrhage 


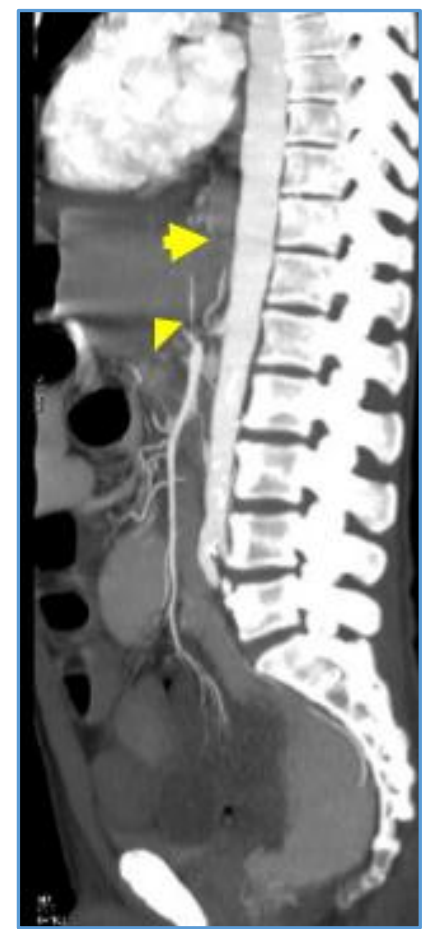

Table/Figure 5. MPR in Sagittal Plane Showing Blocked Celiac Artery and Stenosis of SMA Origin

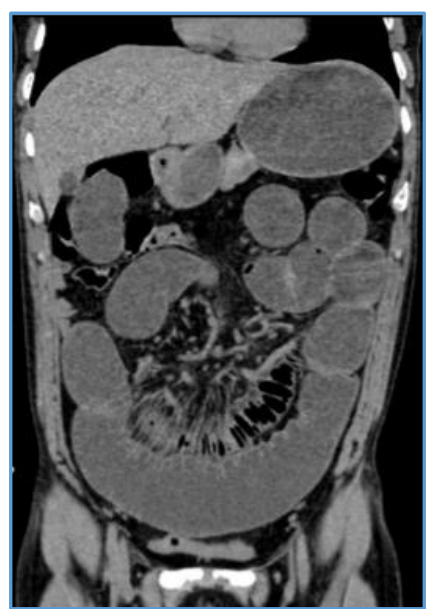

Table/Figure 6. MPR Showing Bowel Distension due to Interruption of Peristalsis Secondary to Ischaemic Insult and Bowel Wall Necrosis in a Case of Acute Mesenteric Ischaemia

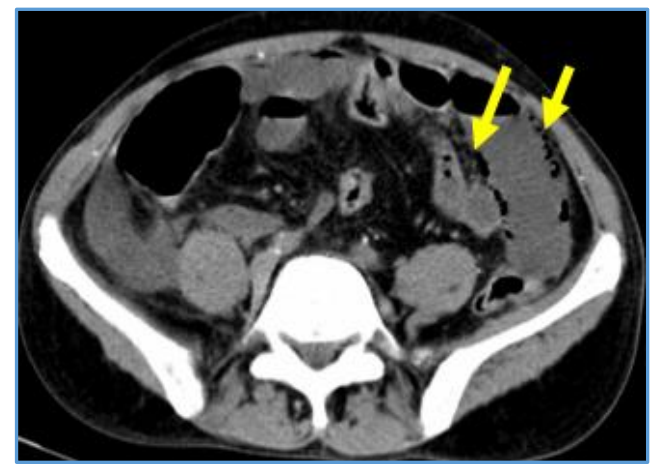

Table/Figure 7. Axial CT Image in a 55-year-old male Showing Pneumatosis - Presence of Air in Bowel Wall because of Transmural Necrosis in Acute Mesenteric Ischaemia

\begin{tabular}{|c|c|c|c|c|}
\hline $\begin{array}{c}\text { CT } \\
\text { Findings }\end{array}$ & $\begin{array}{c}\text { MDCT } \\
\text { Findings in } \\
\text { CMI (22 } \\
\text { Patients) }\end{array}$ & $\begin{array}{c}\text { DSA } \\
\text { Findings } \\
\text { Patients) }\end{array}$ & $\begin{array}{c}\text { Sensitivity } \\
\text { (\%) }\end{array}$ & $\begin{array}{c}\text { Specificity } \\
\text { (\%) }\end{array}$ \\
\hline $\begin{array}{c}\text { Stenosis of } \\
\text { SMA }\end{array}$ & 17 & 16 & 100 & 83.3 \\
\hline $\begin{array}{c}\text { Stenosis of } \\
\text { IMA }\end{array}$ & 12 & 11 & 100 & 90.9 \\
\hline $\begin{array}{c}\text { Stenosis of } \\
\text { celiac trunk }\end{array}$ & 7 & 6 & 100 & 93.7 \\
\hline $\begin{array}{c}\text { Collateral } \\
\text { pathway- } \\
\text { pancreatic } \\
\text { arch }\end{array}$ & 22 & 22 & 100 & 100 \\
\hline $\begin{array}{c}\text { Collateral } \\
\text { pathway- } \\
\text { marginal } \\
\text { artery of the } \\
\text { olon }\end{array}$ & 22 & 22 & 100 & 100 \\
\hline $\begin{array}{c}\text { Table/Figure 8. MDCTA and DSA Correlation } \\
\text { in Patients with Chronic Mesenteric Ischaemia }\end{array}$ \\
\hline
\end{tabular}

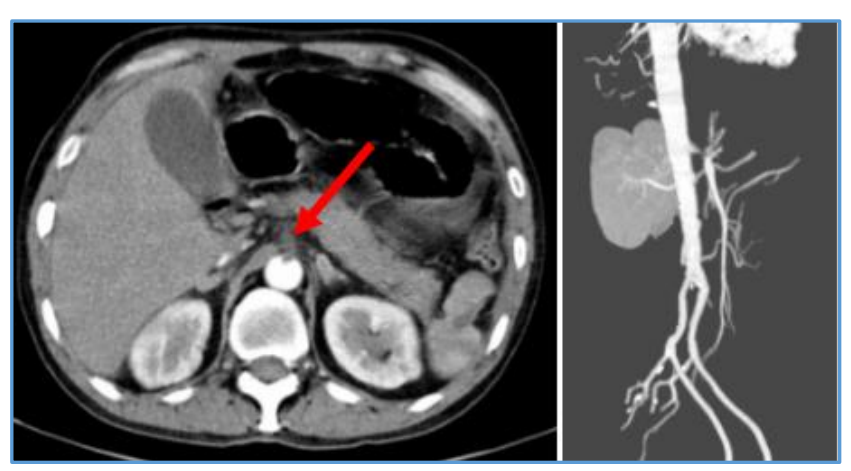

Table/Figure 9. Axial Image Showing Thrombus in Celiac Trunk in Chronic Ischaemia and 3 D Angio Image shows it Very Well

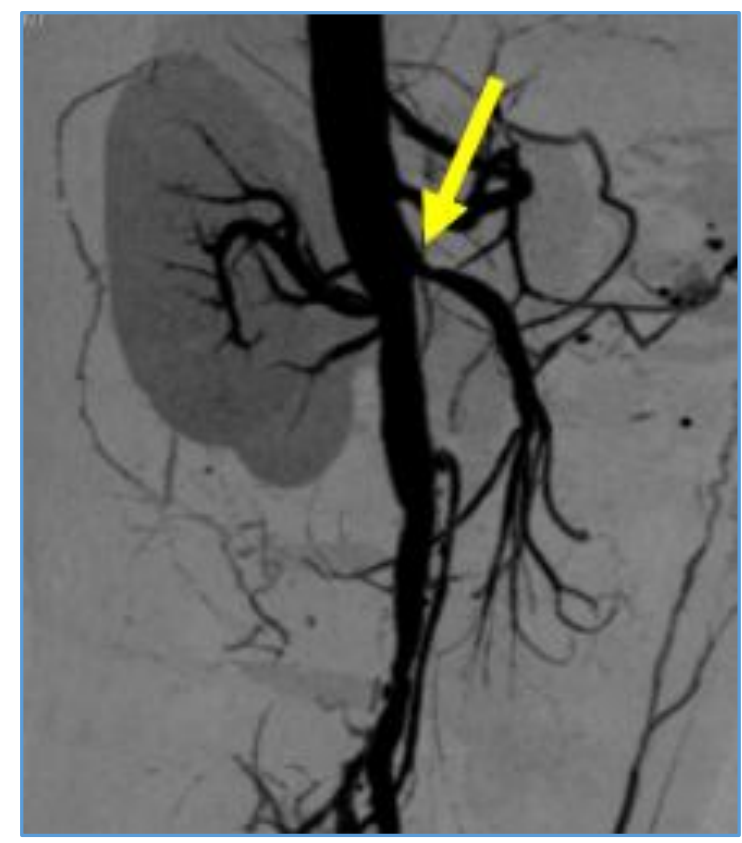

Table/Figure 10. CT Angio Showing Stenosis of Superior Mesenteric Artery in a Patient with Chronic Bowel Ischaemia 


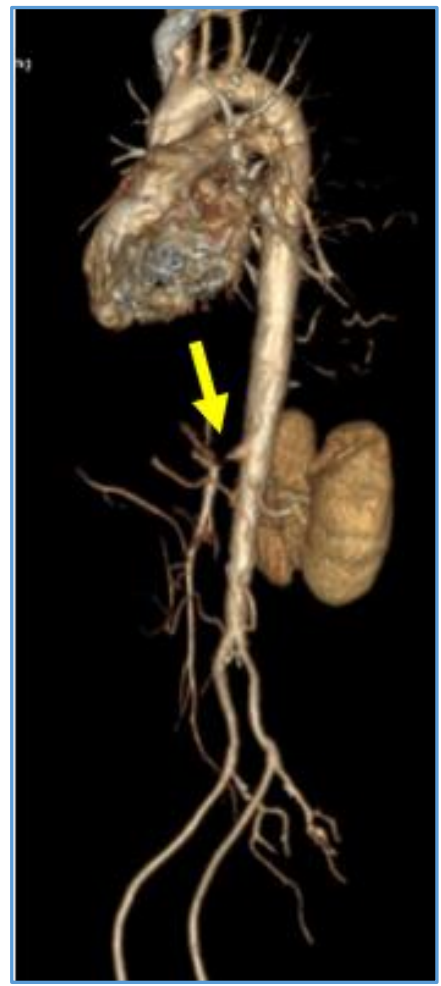

Table/Figure 11. CT angio-Blocked SMA+Celiac and Collaterals Filling Post-block SMA in a 72-year-old Male Patient of Chronic Mesenteric Ischaemia
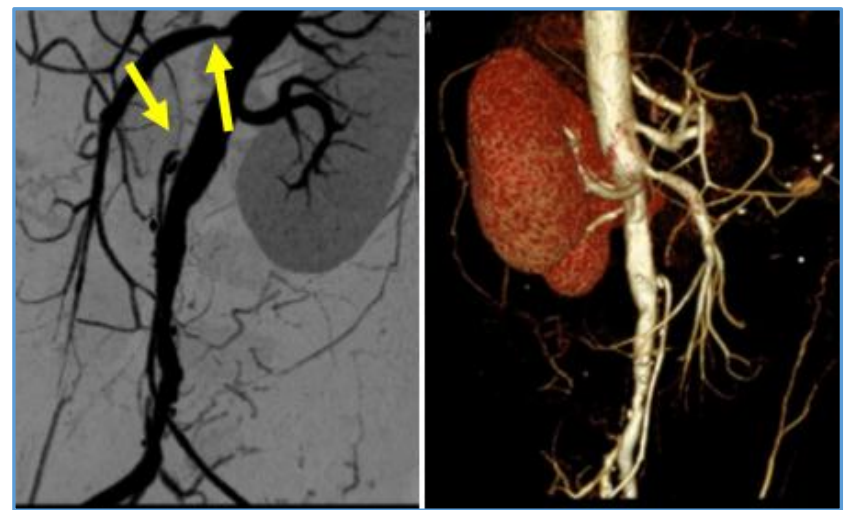

Table/Figure 12. CT angio MIP \& 3-D Angio-stenosis of SMA and IMA with Filling of Collaterals
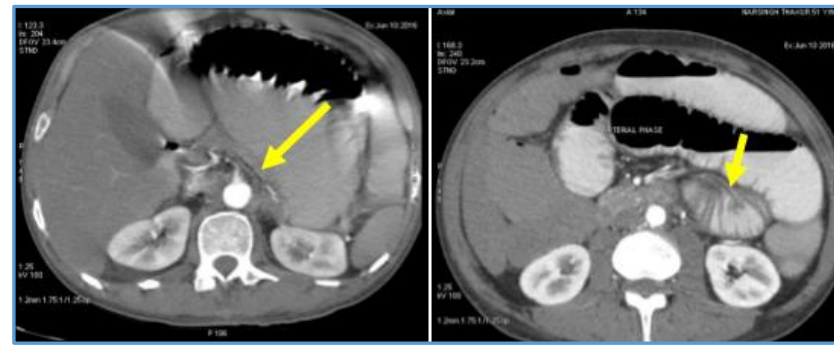

Table/Figure 13. Collateral Vessels, Pancreatic Arch and Marginal Artery of Colon

\section{DISCUSSION}

Acute intestinal ischaemia is an abdominal emergency occurring in nearly $1 \%$ of patients presenting with acute abdomen. The causes can be occlusive or nonocclusive. Early diagnosis is important to improve survival rates. ${ }^{17}$ MDCT examination using IV contrast and multiplanar reconstructions offers precise evaluation of findings in patients suffering with mesenteric ischaemia. Common findings observed are related to bowel wall thickening, distension of bowel and enhancement pattern of bowel wall. Bowel wall thickening was seen in 12 of 15 patients diagnosed as acute mesenteric ischaemia $(80 \%$ of prevalence) while distensions of bowel was found in 53\% of the cases. The bowel wall may appear as low attenuation due to swelling and inflammation or may appear as high attenuation due to haemorrhage in submucosa. Post-contrast examinations show low attenuation of affected segments secondary to compromising of blood flow and with high attenuation due to hyperaemia. ${ }^{17}$

In our series, 7 cases (46.6\%) show high attenuation of bowel wall, which corresponds to hyperaemia suggesting early stage of ischaemic injury. Although seen in wide variety of other pathology like bowel inflammation, these findings are not specific. Furthermore, late consequences of decreased blood flow to bowel wall are more specific to acute mesenteric ischaemia. These findings are related to occlusion of the splanchnic arteries (celiac trunk, SMA and IMA) due to thrombus or dissection.6,9,13 The findings are easily identified during arterial phase of intravenous contrast. ${ }^{8}$

In the acute mesenteric ischaemic group, the dissection of SMA was seen in $33 \%$ of the cases which was an uncommon finding in literature review. ${ }^{18}$ One possible reason of this contradiction is probably due to the small group of patients in our study. SMA thrombosis and thromboembolism were frequently mentioned in literature similar to our series. One case of SMA thrombosis was associated with segmental ischaemia of left kidney which was possibly due to embolic disease.

Multiplanar reformatted images further aided by 3D VRT gives a sensitivity of $96 \%$ and specificity of $94 \%$ according to Kirkpatrick et al. ${ }^{12}$ Necrosis of bowel wall and pneumatosis are very specific late findings seen in severe cases or when study is performed late, however, in most cases the damage is irreversible. $6,9,12$ Similarly, pneumoporta is also a late finding.

Chronic mesenteric ischaemia is a slowly progressing disease process. These cases present as recurrent abdominal pain particularly post meal. Usual cause of CMI is atherosclerotic plaque, which gradually reduces bowel perfusion and these cases develop collateral vessels in order to maintain perfusion of intestinal wall. If there are insufficient collateral vessels then symptoms worsen.7,9

Stenosis of SMA is a common finding $(77 \%)$ in majority of cases of chronic mesenteric ischaemia and is also associated with stenosis of celiac trunk and IMA. Cognet et $\mathrm{al}^{7}$ stated that at least two of these three major arteries should be stenotic as the cause of symptoms. In our series, only 2 symptomatic patients had only one arterial stenosis. Thus, the diagnosis of chronic mesenteric ischaemia is based on the stenosis of artery with associated collaterals in given clinical settings and acute mesenteric findings were excluded. Stenosis of the main vessels (celiac trunk, SMA and IMA) can be very well diagnosed on CT scan.6,7,9 CT findings in chronic mesenteric ischaemia does not demonstrate changes in intestinal loops because of perfusion by collateral vessels. In our study, 22 patients diagnosed with chronic mesenteric ischaemia displayed prominent collaterals at pancreatic duodenal arcade and marginal arteries of the colon. These findings are 
better appreciated on arterial series and on 3D- VRT Images. ${ }^{16}$

Mesenteric venous occlusion comprises up to $10 \%$ of bowel ischaemia cases and is usually associated with mechanical obstruction, but can be due to venous thrombosis. ${ }^{19}$ The later may occur in patients with hypercoagulable syndromes such as sickle cell disease, antiphospholipid antibody syndrome, polycythaemia vera or in hypercoagulable states such as pregnancy and with the use of oral contraceptives. None of cases in our study were diagnosed as mesenteric venous occlusion.

\section{CONCLUSION}

Acute and chronic mesenteric ischaemia is associated with high morbidity and mortality. As the disease mortality and morbidity is very high, and presenting complaints are nonspecific and mimic various general abdomen symptoms, Multiphasic MDCT offers as a self-comprehensive imaging modality of choice for mesenteric ischaemia with excellent evaluation of vascular structures, bowel wall and adjacent mesentery and also demonstrates possible primary cause of mesenteric ischaemia. The specificity of bowel wall thickening pneumatosis and pneumoporta was 100 percent in AMI while in CMI specificity of collateral pathwaypancreatic arch \& marginal artery of the colon was also 100 percent.

\section{REFERENCES}

[1] Bradbury AW, Brittenden J, McBride K, et al. Mesenteric ischemia: a multidisciplinary approach. $\mathrm{Br}$ J Surg 1995;82(11):1446-59.

[2] Mansour MA. Management of acute mesenteric ischemia. Arch Surg 1999;134(3):328-30.

[3] Bassiouny HS. Nonocclusive mesenteric ischemia. Surg Clin North Am 1997;77(2):319-26.

[4] Rhee RY, Gloviczki P. Mesenteric venous thrombosis. Surg Clin North Am 1997;77(2):327-38.

[5] Oliva IB, Davarpanah AH, Rybicki FJ, et al. ACR appropriateness criteria ( $(\mathrm{R})$ ) imaging of mesenteric ischemia. Abdom Imaging 2013;38(4):714-9.

[6] Wiesner W, Khurana B, Ji H, et al. CT of acute bowel ischemia. Radiology 2003;226(3):635-50.

[7] Cognet F, Ben Salem D, Dranssart M, et al. Chronic mesenteric ischemia: imaging and percutaneous treatment. Radiographics 2002;22(4):863-79.
[8] Horton KM, Fishman EK. Volume-rendered 3D CT of the mesenteric vasculature: normal anatomy, anatomic variants, and pathologic conditions. Radiographics 2002;22(1):161-72.

[9] Horton KM, Fishman EK. Multi-detector row CT of mesenteric ischemia: can it be done? Radiographics 2001;21(6):1463-73.

[10] Rha SE, Ha HK, Lee SH, et al. CT and MR imaging findings of bowel ischemia from various primary causes. Radiographics 2000;20(1):29-42.

[11] Martinez JP, Hogan GJ. Mesenteric ischemia. Emerg Med Clin North Am 2004;22(4):909-28.

[12] Kirkpatrick ID, Kroeker MA, Greenberg HM. Biphasic CT with mesenteric CT angiography in the evaluation of acute mesenteric ischemia: initial experience. Radiology 2003;229(1):91-8.

[13] Taourel PG, Deneuville M, Pradel JA, et al. Acute mesenteric ischemia: diagnosis with contrastenhanced CT. Radiology 1996;199(3):632-6.

[14] Shih MC, Angle JF, Leung DA, et al. CTA and MRA in mesenteric ischemia: part 2, normal findings and complications after surgical and endovascular treatment. Am J Roentgenol 2007;188(2):462-71.

[15] Segatto E, Mortele KJ, Ji H, et al. Acute small bowel ischemia: CT imaging findings. Semin Ultrasound CT MR 2003;24(5):364-76.

[16] Horton KM, Fishman EK. Multidetector CT angiography in the diagnosis of mesenteric ischemia. Radiol Clin North Am 2007;45(2):275-88.

[17] Moschetta M, Telegrafo M, Rella L, et al. Multi-detector CT features of acute intestinal ischemia and their prognostic correlations. World J Radiol 2014;6(5):130-8.

[18] Chen JK, Johnson PT, Horton KM, et al. Unsuspected mesenteric arterial abnormality: comparison of MDCT axial sections to interactive $3 \mathrm{D}$ rendering. Am J Roentgenol 2007;189(4):807-13.

[19] Wiesner W, Mortele KJ, Glickman JN, et al. Pneumatosis intestinalis and portomesenteric venous gas in intestinal ischemia: correlation of CT findings with severity of ischemia and clinical outcome. AJR Am J Roentgenol 2001;177(6):1319-23. 